\title{
Image Data Acquisition for NOAA 18 and NOAA 19 Weather Satellites Using QFH Antenna and RTL-SDR
}

\author{
Romi Wiryadinata ${ }^{1}$, Muhammad Khoirussolih $^{1}$, Neneng Rohanah ${ }^{1}$, Imamul Muttakin ${ }^{1}$, \\ Teguh Firmansyah ${ }^{1}$ \\ ${ }^{1}$ Department of Electrical Engineering, Universitas Sultan Ageng Tirtayasa, Cilegon, Indonesia
}

\begin{abstract}
Satellite imaging is widely used in monitoring weather patterns and interpreting satellite images. The limited access to weather satellite image data in real-time is still major issues faced by satellite enthusiasts. NOAA 18 and NOAA 19 weather satellites provide free accessible weather data continuously through Automatic Picture Transmission at $137.9125 \mathrm{MHz}$ and $137.1000 \mathrm{MHz}$ frequencies. In this research the data acquisition system is designed with the help of a Quadrifilar Helix antenna and RTL-SDR. In order to receive both frequencies, the Quadrifilar Helix antenna at $137,5 \mathrm{MHz}$ is designed. Recording, decoding, editing, and viewing of Automatic Picture Transmission signals using WXtoImg software. The result showed that the weather satellite image receptions are sufficiently clear and descriptive, although there are $0.0685 \%$ frequency offsets of Automatic Picture Transmission signal reception on NOAA 18 and $0.0686 \%$ on NOAA 19.
\end{abstract}

\section{Introduction}

Access to obtain local weather satellite image data in real-time is still very limited and difficult to decode the hidden information contained in it. The APT (Automatic Picture Transmission) signal which can be accessed by the public makes NOAA weather satellite an alternative choice to get weather satellite image data. The NOAA 18 and NOAA 19 satellite weather image data will be very useful in GIS (Geographic Information System) that can be used for disaster mitigation, scientific investigation, resource management, development planning, cartography and road planning [1].

The NOAA weather satellite carries five types of sensors, one of which is the AVHRR (Advanced Very High-Resolution Radiometer) [2]. The AVHRR sensor has a function to detect the reflection of electromagnetic waves by clouds, objects on the surface of the earth, as well as the wave of surface emissions of clouds and surface waters. The AVHRR sensor has 6 detectors working on a radiometer channel of different wavelengths ranging from visible and thermal infrared. The APT signals transmitted by NOAA satellites are RHCP (Right Handed Circularly Polarized), so it takes an RHCP type antenna to produce a good signal reception [3][4][5].

\footnotetext{
${ }^{1}$ Corresponding author: wiryadinata@untirta.ac.id
} 
This paper focuses on hardware and software implementation to receive and decode the APT signal on NOAA 18 and NOAA 19 weather satellites. The ideal antenna type for APT receiver at $137 \mathrm{MHz}$ is the QFH antenna (Quadrifilar Helix) [6][7][8]. The QFH antenna we made using copper pipe with the proper dimensions. The presumption that to receive satellite image requires a large parabolic antenna can be broken with a QFH antenna. QFH antennas made with copper pipes are also expected to ease the manufacturing process. SDR is a key area for realizing a variety of software implementations that allow adaptive and reconfigurable communication systems [9].

Many SDR devices used as NOAA weather satellite receivers [10][11][12]. In this research, we use RTL-SDR. This SDR uses Realtek ${ }^{\circledR}$ RTL2832U as the controller and Rafael Micro ${ }^{\circledR}$ R820T2 as the tuner.

\section{Research Method}

The satellite image data acquisition system in this study consists of QFH antenna, LNA, RTL-SDR and a computer that already installed with CubicSDR and WXtoImg. QFH antenna connected at a certain height to receiver and computer shown in Figure 1.

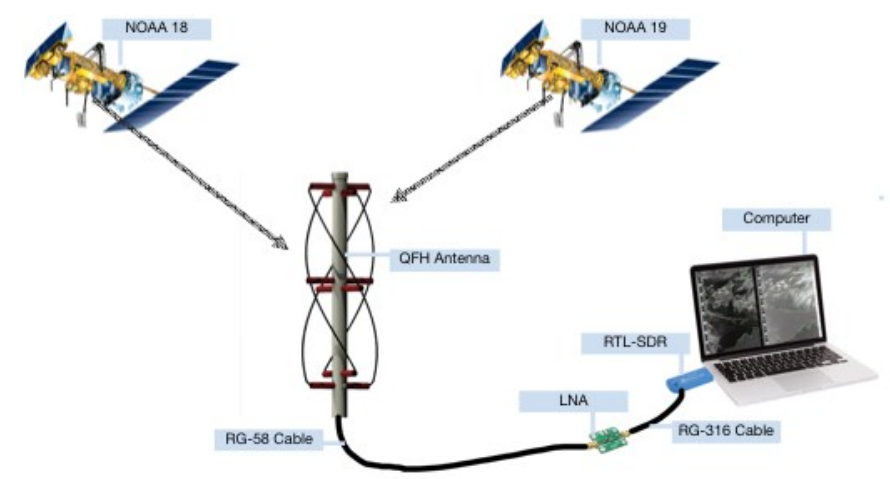

Fig. 1. Simplified Scheme of Satellite Image Data Acquisition System

The diagram block of the satellite image data acquisition system represented by Figure 2. The QFH antenna receives a very weak APT signal from the satellite. We calculated antenna design using QFH calculator [15]. The designed antenna (Figure 4) is a QFH antenna is a $1 / 2$ rotation, 1 lambda wavelength, and $137,5 \mathrm{MHz}$ working frequency. To connect RTL-SDR and QFH antennas required some adapter connectors. Since the RTLSDR device used has an MCX connector, the antenna is using the PL-289 UHF connector and connected to the LNA using an SMA connector. LNA acts as a signal amplifier that amplifies the signal from the QFH antenna.

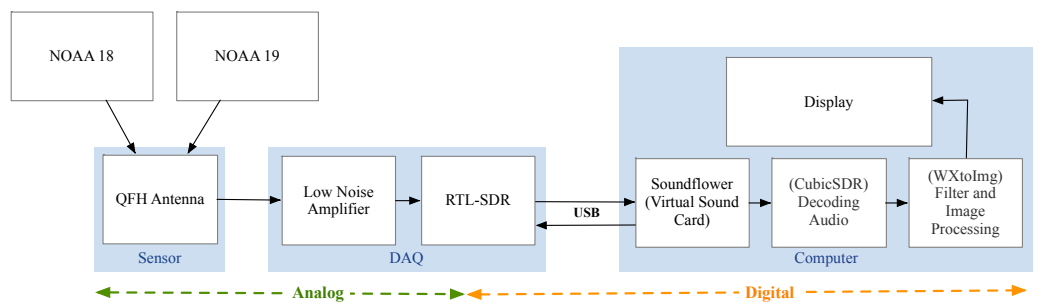

Fig. 2. Block Diagram of Satellite Image Data Acquisition System 

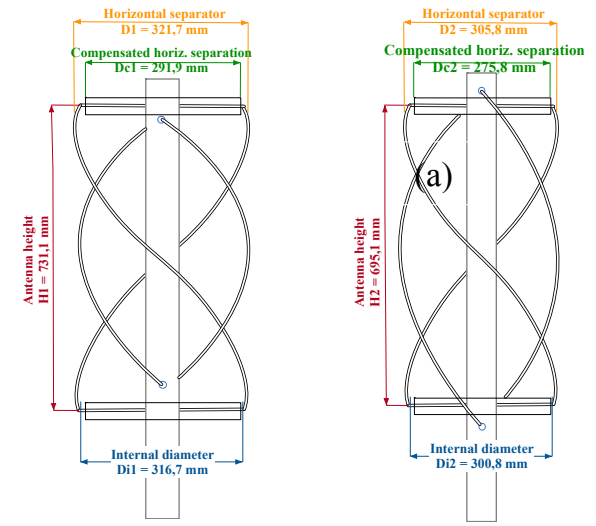

(b)

Fig. 3. QFH Antenna Design. a) Larger Loop, b) Smaller loop

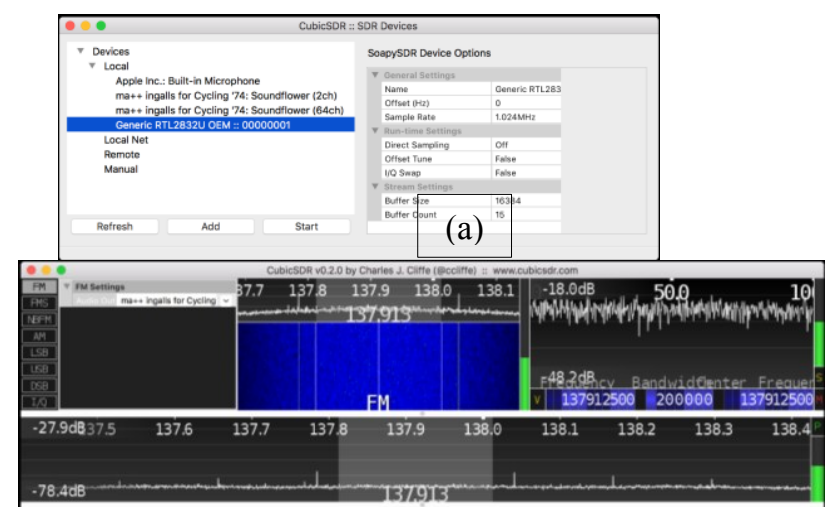

Fig. 4. CubicSDR; a) SDR Device Option and b) Main Window

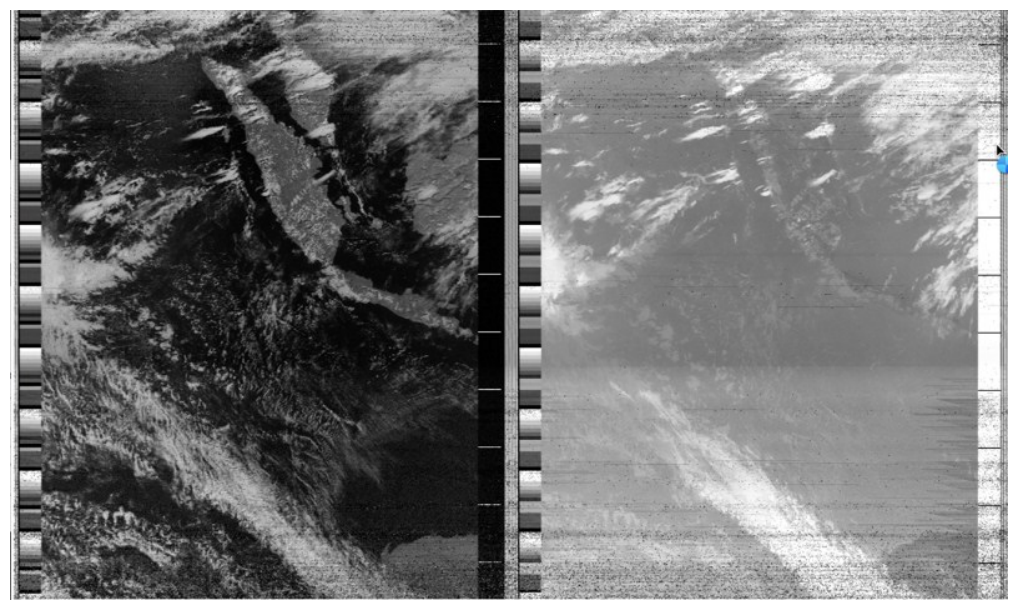

Fig. 5. APT Recording and Decomposition Process on WXtoImg

The information signal received by the QFH antenna is then separated from the carrier signal by RTL-SDR. The RF spectrum was tuned by the tuner on RTL-SDR which is then converted to IF. The demodulator on the RTL-SDR converts from IF to baseband, the ADC digitized the signal at this stage. The CubicSDR software act as the demodulation process control. After opening CubicSDR, SDR RTL2832U device was selected and set sample 
rate of $1024 \mathrm{MHz}$ (Figure 4 (a)). We adjust center frequency of CubicSDR to the NOAA frequency (Fig. 4 (b)), ie 137,9125 for NOAA $18 \mathrm{MHz}$ and 137,1000 MHz for NOAA 19. The signal was passed to CubicSDR and WXtoImg by the Soundflower kernel extension. CubicSDR sets parameters on RTL-SDR and WXtoImg records the received APT signal from RTL-SDR. APT signal captured by QFH antenna, recorded by WXtoImg to generate wav file. WXtoImg is also enabled for decoding and preprocessing APT signals.

\section{Results and Analysis \\ 3.1 QFH Antenna Result}

During the measurement, the antenna is placed in an obstacles free area. The MFj-269 antenna analyzer was used for measurements. Based on the measurement result, the working frequency of $137.5 \mathrm{MHz}$ obtained resistance $41 \Omega$, reactance $10 \Omega$, impedance $42,202 \Omega$, and VSWR 1,3. The following Table 1 results from antenna QFH measurement of a frequency from $132 \mathrm{MHz}$ to $144 \mathrm{MHz}$. The designed QFH antenna meets the specified VSWR specifications, although the best result is shown at $141 \mathrm{MHz}$.

Tabel 1. QFH Antenna Measurement Result

\begin{tabular}{|c|c|c|c|c|c|c|}
\hline $\mathbf{f}(\mathbf{M H z})$ & $\mathbf{R s}(\mathbf{\Omega})$ & $\mathbf{X s}(\mathbf{\Omega})$ & $\mathbf{Z}_{\text {in }}(\mathbf{\Omega})$ & $\mathbf{V S W R}$ & $\boldsymbol{\Gamma}$ & $\mathbf{R L}(\mathbf{d B})$ \\
\hline 132,000 & 39 & 12 & 40,804 & 1,400 & 0,181 & 14,834 \\
\hline 132,500 & 40 & 10 & 41,231 & 1,300 & 0,156 & 16,128 \\
\hline 133,000 & 41 & 9 & 41,976 & 1,300 & 0,139 & 17,128 \\
\hline 133,500 & 42 & 9 & 42,953 & 1,200 & 0,130 & 17,703 \\
\hline 134,000 & 43 & 9 & 43,932 & 1,200 & 0,122 & 18,271 \\
\hline 134,500 & 44 & 9 & 44,911 & 1,200 & 0,115 & 18,820 \\
\hline 135,000 & 44 & 9 & 44,911 & 1,200 & 0,115 & 18,820 \\
\hline 135,500 & 44 & 10 & 45,122 & 1,200 & 0,123 & 18,176 \\
\hline 136,000 & 44 & 10 & 45,122 & 1,200 & 0,123 & 18,176 \\
\hline 136,500 & 44 & 11 & 45,354 & 1,300 & 0,132 & 17,563 \\
\hline 137,000 & 42 & 11 & 43,417 & 1,300 & 0,147 & 16,666 \\
\hline 137,500 & 41 & 10 & 42,202 & 1,300 & 0,147 & 16,656 \\
\hline 138,000 & 40 & 8 & 40,792 & 1,300 & 0,142 & 16,971 \\
\hline 138,500 & 39 & 6 & 39,459 & 1,300 & 0,140 & 17,048 \\
\hline 139,000 & 40 & 2 & 40,050 & 1,200 & 0,113 & 18,917 \\
\hline 139,500 & 41 & 0 & 41,000 & 1,100 & 0,099 & 20,096 \\
\hline 140,000 & 44 & 0 & 44,000 & 1,100 & 0,064 & 23,900 \\
\hline 140,500 & 47 & 0 & 47,000 & 1,064 & 0,031 & 30,193 \\
\hline 141,000 & 51 & 0 & 51,000 & 1,020 & 0,010 & 40,086 \\
\hline 141,500 & 53 & 0 & 53,000 & 1,060 & 0,029 & 30,714 \\
\hline 142,000 & 55 & 4 & 55,145 & 1,100 & 0,061 & 24,302 \\
\hline 142,500 & 53 & 8 & 53,600 & 1,100 & 0,083 & 21,650 \\
\hline 143,000 & 50 & 11 & 51,196 & 1,200 & 0,109 & 19,224 \\
\hline 143,500 & 47 & 12 & 48,508 & 1,200 & 0,127 & 17,954 \\
\hline 144,000 & 44 & 11 & 45,354 & 1,300 & 0,132 & 17,563 \\
\hline
\end{tabular}

Improper antenna impedance has an impact on reducing the efficiency of the antenna because some of the signals are reflected back to the source. The coefficient reflection of $137,5 \mathrm{MHz}$ was 0,147 . Return loss, RL, at $137,5 \mathrm{MHz}$ working frequency obtained value of $16,656 \mathrm{~dB}$. Return loss of $16,656 \mathrm{~dB}$ causes more than $97 \%$ power absorbed to antenna and less than $3 \%$ power reflected. A larger return loss indicates a good impedance matching. 


\subsection{Signal to Noise Ratio}

In this paper, we tested the LNA that connected to QFH antenna with 12 VDC of power, while the RF gain on the RTL-SDR is set at $13 \mathrm{~dB}$. $\mathrm{P}_{\text {signal }}$ is the average power signal received by the antenna. $\mathrm{P}_{\text {noise }}$ is the average value of power noise received by the antenna. The average SNR reading is $57,197 \mathrm{~dB}$.

Tabel 2. SNR of VHF Frequencies

\begin{tabular}{|c|c|c|c|}
\hline Frekuensi $(\mathrm{MHz})$ & $\mathrm{P}_{\text {signal }}(\mathrm{dB})$ & $\mathrm{P}_{\text {noise }}(\mathrm{dB})$ & $\mathrm{SNR}(\mathrm{dB})$ \\
\hline 137,100 & $-2,8409$ & $-60,4167$ & 57,5758 \\
\hline 137,200 & $-2,6515$ & $-60,6061$ & 57,9546 \\
\hline 137,300 & $-2,6515$ & $-60,7955$ & 58,144 \\
\hline 137,400 & $-2,6515$ & $-60,0379$ & 57,3864 \\
\hline 137,500 & $-2,8409$ & $-59,2803$ & 56,4394 \\
\hline 137,600 & $-2,8409$ & $-59,4697$ & 56,6288 \\
\hline 137,700 & $-3,2197$ & $-59,4697$ & 56,25 \\
\hline 137,800 & $-3,0303$ & $-60,6061$ & 57,5758 \\
\hline 137,900 & $-3,4091$ & $-60,2273$ & 56,8182 \\
\hline \multicolumn{3}{|c|}{ Average } & 57,197 \\
\hline
\end{tabular}

The internal amplifier in the RTL-SDR has a noise figure of $<4,5 \mathrm{~dB}$. The amount of noise received causes the signal to degrade, especially when the gain is increased. SNR is a measure of signal quality relative to noise, the higher the better. SNR can be increased by reducing noise from the receiver. The use of LNA can help to reduce the noise effect that can enter through the coaxial cable and help reduce attenuation on a long cable. Apart from the use of LNA, bandwidth can also affect the SNR because the wider the bandwidth of the receiver, the greater the noise generated.

\subsection{Offset Frequncy}

The tested RTL-SDR had low-quality crystal oscillator, with \pm 150 PPM (parts per million) offset. PPM indicates how often the crystal oscillator frequency can deviate from its original frequency. PPM can be changed into percent, 150 PPM equals $0.015 \%$. Referring to $150 \mathrm{PPM}$, there should be $20.687 \mathrm{kHz}$ frequencies offset. Cause signal reception at $137,9125 \mathrm{MHz}$ would be 137,892 MHz. The RF spectrum (Figure 6 (a)) shows the signal at 137.818 MHz which means there are $94.5 \mathrm{kHz}$ frequencies offset or equivalent to $0.0685 \%$. Referring to $150 \mathrm{PPM}$, there should be $20,565 \mathrm{kHz}$ frequencies offset when receiving NOAA 19 satellite signal. Causes signal reception at $137,1000 \mathrm{MHz}$ would be 137.079 MHz. The RF spectrum (Figure 6 (b)) shows the reception of the NOAA 19 satellite signal at $137.006 \mathrm{MHz}$ which means there are of $94 \mathrm{kHz}$ frequencies offset or equivalent to $0.0686 \%$. 


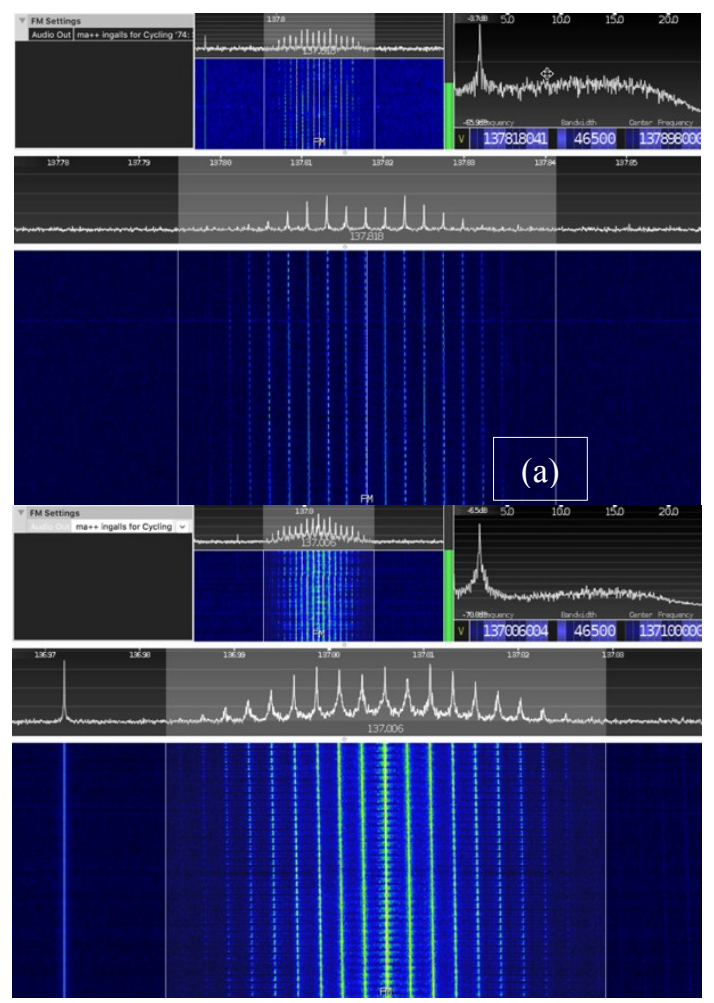

Fig. 6. Offset Frequency on NOAA 18 Satellite Signal Reception, a) 137.818 MHz, b) 137.006 MHz

The frequencies offset on NOAA 18 and NOAA 19 satellite signals reception is not only caused by oscillator crystals but also can depend on environmental factors mainly by temperature. The heating process when a new RTL-SDR is used causes the received frequency signal to deviate over time. The aberrant frequency will be stable after the RTLSDR reaches thermal equilibrium. The solution that can be done is to calibrate the frequency of the CubicSDR application. The frequencies offset can be adjusted by sett the PPM offset value.

\subsection{Acquisition Result}

Data acquisition process begins by sett center frequency on CubicSDR with the bandwidth of $34 \mathrm{kHz}$. The output from CubicSDR needs to be forwarded to WXtoImg so that the recording and decoding of APT signals can be done automatically. Recorded APT signal results are stored automatically via WXtoImg with wav (Waveform Audio File) format. WXtoImg changed the recording of the wav file from the NOAA weather satellite APT signal to image data, which results can be instantly displayed on WXtoImg while still in the recording process. Figure 7 . shows the sample acquisition of NOAA 19 satellite image data in real time on August 15, 2017, at 15:28:44 (UTC+7). The satellite passes from south to north with a maximum elevation angle of $69^{\circ}$ westward. The recording duration lasts for 11 minutes and 45 seconds until 15:40:29 (UTC+7). 


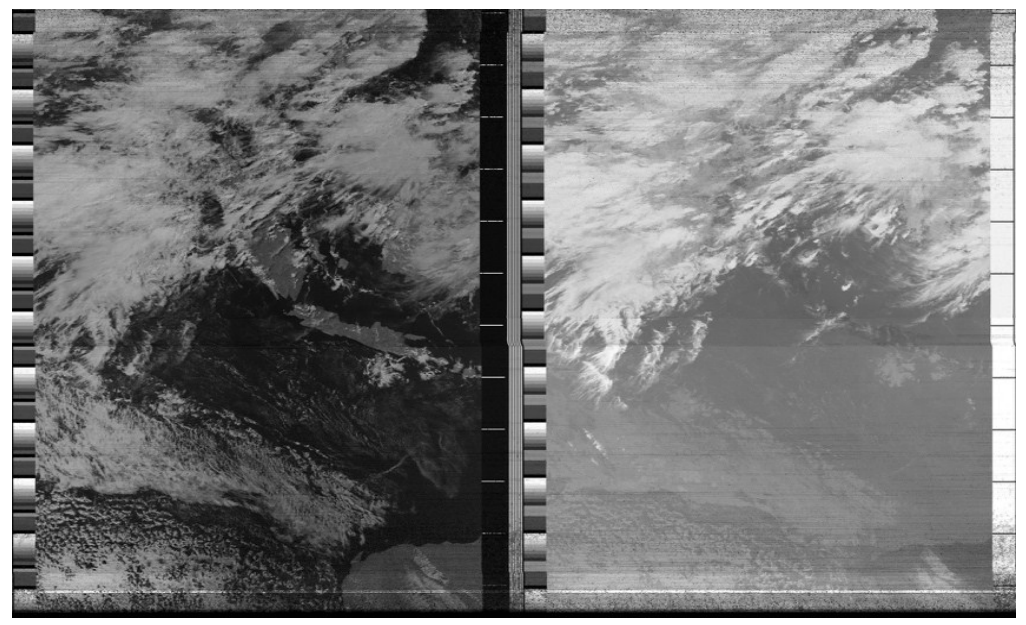

Fig. 7. NOAA 19 On 15 August 2017 at 15:28:44 (UTC+7)

The image data (Figure 7) on the left is the near infrared channel and on the right, is the thermal infrared channel. The image data stored in JPEG format with $2080 \times 1405$ pixels of image resolution. The AVHRR sensor used in NOAA has a very wide field of view with a spatial resolution of $4 \mathrm{~km}$. Spatial resolution refers to the amount of land represented by each pixel. The higher the spatial resolution means the more pixels per unit area. Suitable mapping scales determined from known spatial resolution [15]. Satellites with a spatial resolution of $4 \mathrm{~km}$ produce maps with a 1:8000 of scale.

Samples of image data acquisition when NOAA 18 satellite passes at night on August 15, 2017, at 18:41:32 (UTC +7), shown in Figure 4.11 below. The satellite passes from south to north with a maximum elevation of $52^{\circ}$ to the east. The recording duration lasts for 11 minutes and 27 seconds until 18:52:59 (UTC+7).

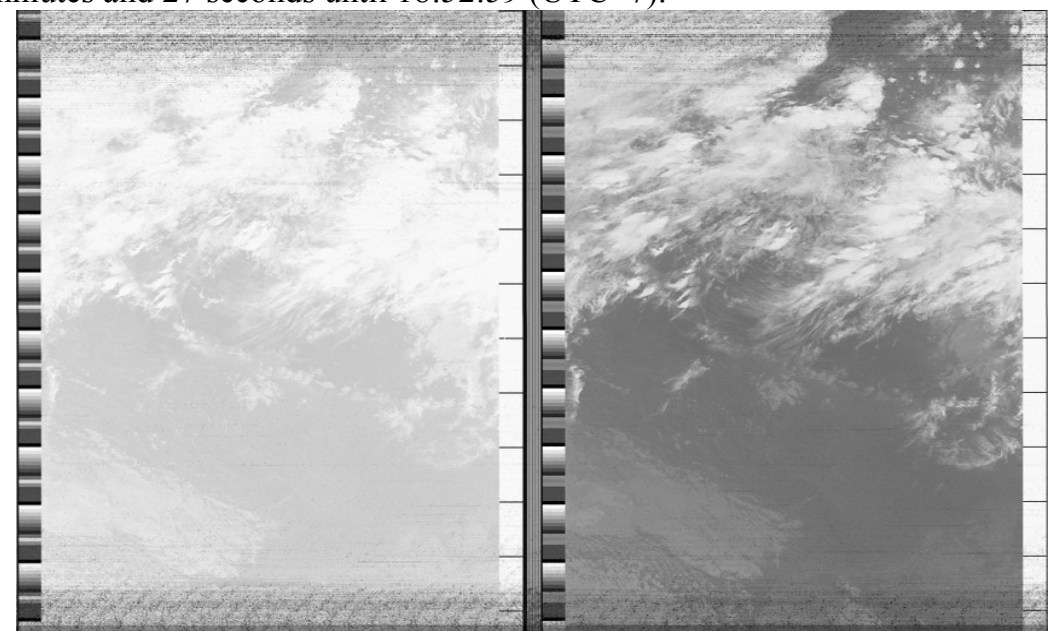

Fig. 8. NOAA 18 On 15 August 2017 at 18:41:32 (UTC+7)

Satellite image data at night shows infrared data on both channels with a different spectrum. Mid-infrared data spectrum on the left is $3.55-3.93 \mu \mathrm{M}$ and thermal infrared on the right is $10.30-11.30 \mu \mathrm{M}$. The image data stored in JPEG format with $2080 \times 1358$ pixels of image resolution. Each channel resolutions are $1040 \times 1358$ pixels. The quality of data reception over analog radio waves affected by unfavorable weather and atmospheres during the trip of APT signals from satellites to ground stations. One of the atmosphere 
effects is energy absorption by each layer of the atmosphere. This absorption depends on the density and air content at the time of the test. There is also an effect of propagation delay and frequency shift, which affected by the ionosphere layer. Disturbances in the ionosphere layer occur when particles ionized, especially by solar radiation. There is always a possibility for improvement in the image data acquisition system, but so far the one we implemented is the best in APT signal reception.

\section{Conclusion}

In this paper, we have built an inexpensive NOAA satellite ground station. The designed QFH antenna was able to work well at $137,5 \mathrm{MHz}$ with 1,3 of VSWR and 42,202 $\Omega$ of impedance, while the best results in $141 \mathrm{MHz}$ with 1,06 of VSWR and $51 \Omega$ of impedance. RTL-SDR is capable of receiving information from NOAA 18 and NOAA 19 satellites by radio waves quite well, although there are $0.0685 \%$ frequency offsets of Automatic Picture Transmission signal reception on NOAA 18 and $0.0686 \%$ on NOAA 19.

\section{References}

1. Wicaksono A., F. F. Muhsoni, and A. Fahrudin, "Aplikasi Data Citra Satelit NOAA-17 Untuk Mengukur Variasi Suhu Permukaan Laut Jawa," J. Kelaut., vol. 3, no. 1, pp. 70-74, (2010)

2. National Oceanic and Atmospheric Administration, User's Guide for Building and Operating Environmental Satellite Receiving Stations, no. February. Suitland,: U.S. DEPARTMENT OF COMMERCE, (2009)

3. Gao S.S., Q. Luo, and F. Zhu, Circularly Polarized Antennas. Chichester: John Wiley \& Sons, (2014)

4. Stutzman W.L. and G. A. Thiele, Antenna Theory and Design 2nd edition. Hoboken: John Wiley \& Sons, (1998)

5. Balanis C.A., Antenna Theory Analysis and Design 3rd Edition. Hoboken: John Wiley \& Sons, (2005)

6. Hollander R.W., Resonant Quadrafilar Helical Antenna. Den Haag: Working Group Satellites, (1999)

7. Fusco V.F., R. Cahill, and R. L. Li, "Quadrifilar loop antenna," IEEE Trans. Antennas Propag., vol. 51, no. 1, pp. 115-120, (2003)

8. Govarthanam K.S., M. Abirami, and J. Kaushik, "Economical Antenna Reception Design for Software Defined Radio using RTL-SDR," in Proceedings of the International Conference on Innovative trends in Electronics Communication and Applications, 2014, pp. 185-190.

9. Stewart R.W., K. W. Barlee, D. S. W. Atkinson, and L. H. Crockett, Software Definded Radio using MATLAB \& Simulink and the RTL-SDR. Glasgow: Strathclyde Academic Media, (2015)

10. Patil C., T. Chavan, N. Jhurani, D. Jethani, and M. Chaudhari, "Satellite Image Acquisition Using SDR,” Int. J. Innov. Res. Comput. Commun. Eng., vol. 4, no. 6, pp. 2257-2263, (2016)

11 Mahmood S., M. T. Mushtaq, and G. Jaffer, "Cost Efficient Design Approach for Receiving the NOAA Weather Satellites Data," in Proceedings of the IEEE Aerospace Conference, pp. 1-6, (2016)

12. Hassini A. and A. H. Belbachir, "Hardware and software consideration to use near real time MSG-SEVIRI and NOAA-AVHRR images," c, ISPS 2013, pp. 12-16, (2013)

13. Maheshwarappa M.R. and C. P. Bridges, "Software defined radios for small satellites," Proc. 2014 NASA/ESA Conf. Adapt. Hardw. Syst. AHS 2014, no. July 2014, pp. 172-179, (2014)

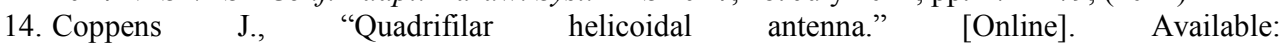
http://jcoppens.com/ant/qfh/calc.en.php. [Accessed: 14-Apr-2017].

15. Arlinghaus S.L. and J. J. Kerski, Spatial Mathematics: Theory and Practice through Mapping 1st Edition. Boca Raton: CRC Press, (2014) 